\title{
Mutations of PAX3 unlikely in Waardenburg syndrome type 2
}

Sir - Waardenburg syndrome has been divided with regard to dystopia canthorum (DC), into types 1 (presence) and 2 (absence) of the sign'; this can be confidently assessed with index W (ref. 2). Lack of penetrance for DC in WS1 occurs in no more than $1 \%$ of cases. Most clinically 'nonpenetrant' cases are those with nonapparent dystopia canthorum $(\mathrm{NAD})^{2}(1.86<\mathrm{W}<2.07)$ who appear to be 'normal'. Family WS.15 in Tassabehji et $a l^{3}$, purported to be an example of WS2, is instead a typical case of WS1 (I-2, Fig. 4, left; W=2.23). I-2 has a nonpenetrant son (II-2, Fig. 4 , right; $W=1.83$ ) very similar to the mother in family 7 of Waardenburg's original paper ${ }^{4}$, the first and almost single published case of nonpenetrance for DC in WS1 (if stringent biometic criteria are applied $)^{2,5}$. Individual III-2 (W=2.05), grandson of I-23, is a case of NAD according to definition ${ }^{2}$.

It is generally and clinically important to establish that PAX3 mutations (besides those in alveolar rhabdomyosarcoma ${ }^{6}$ ) are associated only with WS1 and not WS2, as seems to be the case. The type 1 (WS1) family supposedly not showing linkage to ALPP was, however, not excluded from the 2q35-q37 region? On the other hand, at least two out of three WS2 families (none biometrically documented: without stated $W$ index values) did not show linkage to $A L P P$, and furthermore, were heterogeneous $(\mathrm{P}=0.006)$ with respect to WS1 (ref. 7).

Thus, PAX3 does not seem to be mutated in Waardenburg syndrome type 2 , according to the available biometric $^{3}$ and linkage data ${ }^{7}$.

Sergio Arias

Laboratory of Human Genetics,

Apartado 21827,

Caracas 1020A,

Venezuela

In Reply - The main point of our paper was to show that loss-of-function mutations in $P A X 3$ could cause Waardenburg syndrome (WS). The small family to which Arias refers included one affected person without dystopia $(\mathrm{W}=1.83)$, one with mild dystopia ( $W=2.21)$ and one with $\mathrm{W}=2.05$, an intermediate figure below the diagnostic threshold $(\mathrm{W}=2.07)$ of the Waardenburg Consortium. One could argue about diagnostic labels and whether they apply to individuals or to families; as an individual our proband was type 2; the family as a whole was neither typical type 1 or type 2 . Any classification based on presence or absence of one of the many manifestations of this variable disease is bound to be fallible. However, molecular analysis has in general supported the distinction, first made by Arias himself, between type 1 and type 2 WS. So far, no family with unambiguous type 2 WS has been shown to have a mutation in $P A X 3$, although there are now data showing that in some families unambiguous type 1 WS does not map to the location of PAX3.

Andrew P. Read

Department of Medical Genetics,

St Mary's Hospital,

Manchester M13 0JH, UK

\section{Valerie Newton}

Centre for Audiology,

University of Manchester,

Manchester M13 9PL, UK

\section{Michael Patton}

SW Thames Regional Genetics Service,

St. George's Hospital Medical School, London SW17 0RE, UK

\footnotetext{
References

1. Arias, S. Birth Def. 7, 87-101 (1971).

2. Arias, S. \& Mota, M. J. Genet. Hum. 26, 103-131 (1978).

3. Tassabehii, M. ot a. Nature Genet. 3, 26-30 (1993)

4. Waardenburg, P.J. Am. J. hum. Genet. 3, 195253 (1951).

5. Arias, S.Am. J. med. Genet. 17, 863-865(1984).

6. Barr, F.G. et al. Nature Genet. 3, 113-117(1993)

7. Farrer, L.A. et al. Am. J. hum. Genet. 50, 902-
} 913 (1992).

\section{Anticipation in spinocerebellar ataxia type 2}

Spinocerebellar ataxias (SCAs) are characterized by a progressive degeneration of specific neurons of the cerebellar cortex, deep cerebellar nuclei, brain stem and spinal cord. Affected individuals suffer from severe ataxia, dysarthria and variable degrees of motor disturbance and neuropathy. The gene for one subtype of the dominantly inherited ataxias (SCA1) maps to chromosome 6P (refs 1,2 ), and a second subtype (SCA2) was recently mapped to chromosome $12 \mathrm{q}$ between $D 12 S 58$ and $P L A 2$, in a large pedigree of Cuban descent ${ }^{3}$. We have now identified a second pedigree with linkage to $12 \mathrm{q}$ and established closer flanking markers for SCA2. In contrast to the Cuban pedigree this pedigree reveals a remarkable degree of anticipation of disease onset. Given the recent identification ${ }^{2}$ of an expanded CAG triplet repeat in SCA1, this finding suggests a similar mechanism may underlie at least some cases of SCA2 as well.

The FS pedigree is of Southern Italian descent and segregates SCA in five generations (Fig. 1a). All affected individuals show marked appendicular and gait ataxia as well as slow saccadic eye movements ${ }^{4}$. DNA was available from 60 individuals including 7 affecteds. Mean age of onset in 19 affecteds was $26.9 \pm 12.5$. We first excluded linkage to $S C A 1$ based on the observation of multiple obligate recombinants between the disease phenotype and D6S89 and D6S109, which are known to flank the SCA1 locus ${ }^{2}$. Multipoint analysis excluded the mutation in this family from a $15 \mathrm{cM}$ region including the region between D6S89 and D6S109. To determine whether this family has 